\title{
Patient Reported Outcome Measure (PROM) of Quality of Life After Prostatectomy - Results from a 5-Year Study
}

\author{
Liselotte Jakobsson* and Per Fransson
}

School of Health and Society, Kristianstad University, SE-291 88 Kristianstad, Sweden

\begin{abstract}
Prostate cancer is the most common cancer among men in Sweden, and treatment is negatively affecting the patients' quality of life. Even so, long term experiences are sparse and implications for nursing practice are little known. The aim of this study was to determine areas of functioning and factors impacting quality of life, QOL, during and five years after radical prostatectomy (RP) using a quality of life questionnaire and a specific module for prostate cancer. A longitudinal study was performed with consecutively included Swedish men from baseline and after RP treatment $(\mathrm{n}=222)$ from 2003 to 2011 to obtain their opinions on quality of life. Data was gathered through a mail out - mail in procedure at baseline, 3 months, $1-3$ and 5 years after treatment with a response rate of $94.14 \%-75.2 \%$. One reminder was sent on each occasion. Identified areas with increased functioning after five years were emotional and social functioning. QOL ratings did not change over the years. Sexual activity and functioning decreased and hormonal treatment-related symptoms increased. Impact on QOL was found regarding emotional and social functioning, nausea/vomiting, pain and hormone-related symptoms. Increasing age, living with a partner and educational level had no significant impact on QOL. Implications for nursing are to initially focus on physical problems and at times for follow-up visits pay attention also to emotional and social aspects of life. To be able to make a difference in the patient's life, nurses need to bridge the gap between in-hospital treatment and everyday life outside hospital.
\end{abstract}

Keywords: Patient reported outcome measure PROM, quality of life, quantitative longitudinal study.

\section{INTRODUCTION}

Prostate cancer is the most frequent cancer among men in Sweden, representing $32.2 \%$ of all cancer forms, with an annual increase of $0.7 \%$ in the last 10 years [1]. The total five-year survival rate of prostate cancer was $87.3 \%$ in 2009 [2], which means that most men survive cancer and treatment. In Sweden, there is a consensus about nonscreening, but in a long-term study over 15 years BillAxelsson et al. found that a localized and well differentiated tumour treated with radical prostatectomy, RP, versus watchful waiting, reduced the rate of deaths by $14.6 \%$ compared to $20.7 \%$ respectively [3]. This implies that more lives may be saved by detection and treatment. International research has shown [4] that even if life is prolonged, problematic symptoms are presented over the years following treatment.

The most common treatment options offered to men are radical prostatectomy, external beam radiation, brachytherapy and active surveillance. For the patients, it may be difficult to choose between treatment options, especially for those with an early-stage prostate cancer [5], since all treatments have side effects such as bowel problems connected with beam radiation [6] and radical prostatectomy with urinary and sex life problems [7]. Symptoms following radical prostatectomy may overlap general ageing problems such as fatigue, decreased mobility and insomnia. Fatigue is

*Address correspondence to this author at the School of Health and Society, Kristianstad University, SE-291 88 Kristianstad, Sweden; Tel: 46442040 25; Fax: 46442040 43; E-mail: Liselotte_Jakobsson@Hkr.se a well-known general adverse treatment-related side effect of cancer and, Fransson found increased problems with fatigue from pre-treatment up to five years post radiation treatment in a sample of men with prostate cancer $(n=407)$. If fatigue is presented to a similar degree in men treated with RP was not investigated. Impaired mobility and insomnia are phenomena described in relation to cancer illnesses and old age, and Stenholm et al. found impaired mobility correlating to insomnia in a Finnish population study of 2825 men of 55 years of age and older [8]. The authors, however, did not clearly differentiate between possible origins, and there are national differences between countries. Treatment options for prostate cancer are continually improving, and whether a south Swedish sample presents similar problems is not known.

Quality of life is described by Aaronson et al. as a multidimensional concept that covers life functions, specific symptoms and overall global health [9]. Although referring to patients with cancer, the core concept is general and gives a subjective view on persons' life quality. The underlying assumption is that there may be a presence of symptoms and still high ratings for quality of life. Quality of life assessments are often used as tools for predicting and describing treatment outcomes and how life is perceived after treatment. It has been shown earlier that high ratings for quality of life may follow in the wake of cancer treatment [10].

Treatment affects quality of life in patients with cancer. Abraham et al. found that most men 93\% ( $\mathrm{n}=1$ 542) were satisfied with their choice of treatment (prostatectomy) 
indicating a non-significant trend toward time dependence, i.e. that symptoms of illness decreased over time [11]. Shortterm satisfaction was connected to e.g. post-surgery symptoms such as indwelling urinary catheter treatment and long-term satisfaction, e.g. long time after surgery, was more often connected to urinary and sexual function [12]. Since the treatment choice affects life and quality of life for the rest of the men's lives, it is a challenging task for the health professions to monitor and handle cancer and treatment sequels according to evidence based medicine.

\section{AIM}

The aim of our study was in threefold, including 1) identifying change in domains of functioning in patients from baseline to five years after treatment, and 2) identifying important factors influencing their QOL ratings over five years measured with the EORTC QLQ C-30 and PR-25, and 3 ) investigating the impact of increasing age, unemployment $v s$ employment and marital status (having a partner) on their quality of life across time.

Specific research questions answering the objectives:

1. ${ }^{1}$ What aspects on quality of life $^{2}$ as assessed by the EORTC QLQ C-30 show significant change from baseline to five years, and what aspects from the PR25 ?

2. What aspects of functioning and symptoms from the EORTC QLQ C-30 and the PR-25 influence quality of life ${ }^{3}$ ratings over the years?

3. Is there an effect on quality of life ${ }^{3}$ at five years from increasing age, unemployment $v s$ employment and whether or not living with a partner?

\section{MATERIAL AND METHODOLOGY}

\section{Study Design}

A prospective, longitudinal survey design was used, and data was collected at six points: pre-treatment (baseline), 3 months after RP, 1-3 and 5 years after RP.

\section{Participants}

Inclusion criteria included Swedish-speaking men diagnosed with localized prostate cancer (LPC), regardless of age, with no signs of impaired brain function and eligible for radical prostatectomy between the years 2000 and 2007, and referred to a department of Urology in southern Sweden. The men were consecutively asked to participate in the longitudinal study. A total of 222 men accepted. By the end of the data collection period, December 2011, a total of 167 men remained in the study. The clinical treatment procedure consisted of radical retropubic prostatectomy in all cases with a nerve-sparing technique where appropriate and possible.

\section{Data Collection}

During the recruitment period, consecutive eligible patients were verbally invited to participate in the study by a specialist urological nurse. A consent form and the first

\footnotetext{
${ }^{1}$ Figures refer to the result section.

${ }^{2}$ The concept of quality of life.

${ }^{3}$ The index of quality of life.
}

questionnaire (baseline) were handed out to all the participating patients. The patients were asked to complete the baseline questionnaire at home and a pre-paid return envelope accompanied the questionnaire. On the following data collection occasions, the questionnaires and pre-paid return envelopes were sent by mail-out-mail-in procedure and this was repeated at 3 months, 1,2,3 and 5 years.

\section{Questionnaires}

To determine factors under study, the Health-Related Quality of Life questionnaires, QLQ C-30 and the Prostate cancer specific, QLQ PR-25 were used for assessment. The questionnaires were developed for measurement of quality of life in cancer patients [9], and for specific prostate cancer symptoms [13], respectively, where at least a 10 point difference in scoring is clinically interesting [14]. The 30 variables of the QLQ C-30 explore physical, emotional, cognitive, role and, social functioning and quality of life, all arranged in a Likert scale format. Also included are multiitem variables of fatigue, nausea/vomiting and pain, together with single items assessing dyspnoea, appetite loss, constipation, financial impact and diarrhoea. All variables are transformed into scales and further into separate indexes and, all indexes are summarized into a concept of quality of life. Chronbahs' alpha has been described between 0.52 to 0.89 for the instrument [9]. The prostate-specific module, PR-25, is developed for prostate cancer and is to be used in connection with the QLQ C-30. The instrument consists of 25 variables in Likert scale format. The variables assess sexual activity and functioning, urinary, bowel and hormonal treatment-related problems and the frequency of using incontinence aids. All variables are transformed into scales and further into separate indexes and, all indexes are summarized into a health status presenting life with prostate cancer. Chronbachs' alpha has been described between 0.30 to 0.86 for the instrument [13]. Raw scores for the QLQ C30 and the PR-25 were transformed into a 100-point scale [15], where functional scales with high ratings represent high functioning, except for scales of fatigue, pain and, nausea/vomiting where low scores represent a low grade of problems, i.e. a high grade of functioning. For single item scores, lower scores represent better functioning, i.e. few symptoms and problems.

\section{Statistical Analysis}

To answer study-specific questions $(1,2,3)$ the following statistical procedures were performed:

1. Differences in categorical data, both in the nominal and ordinal scale levels, were tested with the Chisquare test. Differences in mean ratings of all variables across the data collection period (five years) were tested with one-way analysis of variance. When significant ANOVA was found, the Bonferroni post hoc conservative analysis was applied due to rather small subgroups to avoid mass significance $[16,17]$. Pearson's correlation was applied to determine associations between demographic data, numerical variables and, between the questionnaire variables under study.

2. The outcome on QOL ratings from the questionnaires was determined using a multiple regression analysis 
model. The analysis model explains how much of the variation in QOL ratings can be explained by the variables, and the contribution of each questionnaire variable. The test uses a quantitative dependent variable (QOL) and predicts the influence from one or more independent variables. In this study, all variables were included in the model (enter method) except two from PR-25, due to the requirement of not using highly correlated variables $(>0.7-0.9)$ in the analysis [18]. Thus, the created model estimated the degree to which QOL variation was explained by the subscales in the model. Before creating a model, a correlation analysis was done and relationships among the variables established. Correlations did not exceed 0.7-0.9 in a bivariate correlation, thus indicating no multicollinearity. The multicollinearity was further checked for by collinearity diagnostics where a tolerance value $>0.1$ and a VIF (Variance inflation Factor) value $<10$ indicated no multicollinearity, i.e. a linear correlation that makes it possible to distinguish between variable influences [19].

3. Whether there was an influence on quality of life in general, from increasing age, unemployment $v s$ employment and whether or not living with a partner at five years was tested by a logistic regression analysis. Early or late symptoms was used as the dependent dichotomised variable (baseline $=0,5$ years $=1$ ). The model was tested by the HosmerLemeshow goodness-of-fit test. Cox and Snell R Square and Nagelkerke R Square values indicated the amount of variation in the dependent variables that was explained by the model [18]. All questionnaire variables were included in our study model, together with age (continuous variable), living with a partner $($ no $==0$, yes $=1)$, still working or not $($ no $=0$, yes $=1)$.

The study was approved by the Ethical Committee, The medical Faculty at Lund University (U451-01).

\section{RESULTS}

At baseline, 222 men accepted to participate in the study. Of the 222 men, 167 (75.2\%) men were still included in the study after five years (Table $\mathbf{1}$ ).

\section{Patient Characteristics}

The study participants were aged 36 to 75 years, most men were married 181/222 (82.0\%) and 93/222 (42.0\%) had an educational level of post-compulsory school. Some men were retired, 86/222 (39.0\%) while 56/222, (25.2\%), were still in active employment.

\section{Changes in Quality of Life from Baseline to Five Years}

\section{Health-Related Quality of Life, EORTC QLQ C-30}

Results for the QLQ C-30 scale (Table 2) show physical, role and cognitive functioning scales rated as highly functioning at baseline and at 3 months (mean 96.3-90.4) and at five years with no fluctuation over the years $(\mathrm{p}=0.11$ 0.98). Emotional functioning showed a low degree of functioning at baseline, (mean 78.9) and a significant increase over the years to 90.9 in year five $(p=<0.00)$. Social
Table 1. Characteristics of Participants at Baseline $(n=222)$

\begin{tabular}{|c|c|}
\hline \multicolumn{2}{|l|}{ Variables } \\
\hline \multicolumn{2}{|l|}{ Age } \\
\hline Year, m, (sd) & $62.7(6.09)$ \\
\hline Range & $36-75$ \\
\hline \multicolumn{2}{|l|}{ Living with partner ${ }^{l)}$} \\
\hline Yes & 181 \\
\hline No & 19 \\
\hline \multicolumn{2}{|l|}{ Educational level } \\
\hline$<$ Compulsory school & - \\
\hline Compulsory school & 93 \\
\hline Post-Compulsory below University level & 48 \\
\hline University level & 25 \\
\hline Others & 34 \\
\hline \multicolumn{2}{|l|}{ Employment status } \\
\hline Working & 56 \\
\hline Retired & 86 \\
\hline On sick leave & 2 \\
\hline Disability pension & 6 \\
\hline Others & 7 \\
\hline \multicolumn{2}{|l|}{ Tumour stage } \\
\hline $\mathrm{T} 1 \mathrm{~B}$ & $2(1 \%)$ \\
\hline $\mathrm{T} 1 \mathrm{C}$ & $44(65 \%)$ \\
\hline $\mathrm{T} 2$ & $73(33 \%)$ \\
\hline T3 & $3(1 \%)$ \\
\hline \multicolumn{2}{|l|}{ Gleason score } \\
\hline 3 & $5(2 \%)$ \\
\hline 4 & $24(11 \%)$ \\
\hline 5 & $40(18 \%)$ \\
\hline 6 & $80(36 \%)$ \\
\hline 7 & $60(27 \%)$ \\
\hline 8 & $9(4 \%)$ \\
\hline 9 & $2(1 \%)$ \\
\hline \multicolumn{2}{|l|}{$P S A$} \\
\hline Mean & 7.7 \\
\hline Range & $0.4-34.0$ \\
\hline \multicolumn{2}{|l|}{ Participants (n) } \\
\hline Baseline & 222 \\
\hline 3 months & 209 \\
\hline 1 year & 197 \\
\hline 2 years & 194 \\
\hline 3 years & 187 \\
\hline 5 years & $167(75.2 \%)$ \\
\hline
\end{tabular}


Table 2. Questionnaire Ratings at Baseline, 3 Months, 1-3 Years and 5 Years

\begin{tabular}{|c|c|c|c|c|}
\hline QLQ C-30 Scale & $n^{a}$ & Mean & SEM & $\begin{array}{l}\text { p-Value } \\
\text { (ANOVA) }\end{array}$ \\
\hline \multicolumn{5}{|c|}{ Physical functioning } \\
\hline Baseline & 197 & 96.3 & 0.73 & \\
\hline 3 months & 204 & 94.9 & 0.80 & \\
\hline 1 year & 197 & 95.1 & 0.80 & \\
\hline 2 & 194 & 95.3 & 0.71 & \\
\hline 3 & 187 & 94.4 & 0.77 & \\
\hline 5 years & 166 & 93.9 & 0.89 & 0.109 \\
\hline \multicolumn{5}{|l|}{ Role functioning } \\
\hline Baseline & 199 & 92.2 & 1.41 & \\
\hline 3 months & 206 & 91.3 & 1.30 & \\
\hline 1 year & 195 & 94.5 & 1.18 & \\
\hline 2 & 192 & 95.8 & 0.95 & \\
\hline 3 & 186 & 93.4 & 1.12 & \\
\hline 5 years & 166 & 94.5 & 1.09 & 0.227 \\
\hline \multicolumn{5}{|c|}{ Emotional functioning } \\
\hline Baseline & 199 & 78.9 & 1.37 & \\
\hline 3 months & 206 & 87.0 & 1.11 & $0.000 *$ \\
\hline 1 year & 196 & 89.8 & 1.08 & \\
\hline 2 & 193 & 89.1 & 1.16 & \\
\hline 3 & 186 & 89.2 & 1.07 & \\
\hline 5 years & 166 & 90.9 & 1.06 & $0.000 *$ \\
\hline \multicolumn{5}{|c|}{ Cognitive functioning } \\
\hline Baseline & 199 & 90.1 & 1.03 & \\
\hline 3 months & 208 & 90.4 & 0.85 & \\
\hline 1 year & 197 & 90.0 & 0.97 & \\
\hline 2 & 193 & 89.7 & 0.99 & \\
\hline 3 & 186 & 89.4 & 1.01 & \\
\hline 5 years & 167 & 90.3 & 1.09 & 0.979 \\
\hline \multicolumn{5}{|l|}{ Social functioning } \\
\hline Baseline & 196 & 92.4 & 1.16 & \\
\hline 3 months & 208 & 86.3 & 1.31 & $0.001 *$ \\
\hline 1 year & 196 & 88.1 & 1.42 & \\
\hline 2 & 192 & 91.0 & 1.19 & \\
\hline 3 & 186 & 90.8 & 1.64 & \\
\hline 5 years & 165 & 92.2 & 1.22 & $0.003 *$ \\
\hline \multicolumn{5}{|c|}{ QOL, Global health status } \\
\hline Baseline & 198 & 78.1 & 1.43 & \\
\hline 3 months & 208 & 77.0 & 1.37 & \\
\hline 1 year & 196 & 79.6 & 1.34 & \\
\hline 2 & 191 & 81.6 & 1.19 & \\
\hline
\end{tabular}

(Table 2) contd......

\begin{tabular}{|c|c|c|c|c|}
\hline QLQ C-30 Scale & $\mathbf{n}^{\mathrm{a}}$ & Mean & SEM & $\begin{array}{c}\text { p-Value } \\
\text { (ANOVA) }\end{array}$ \\
\hline 3 & 185 & 81.2 & 1.30 & \\
\hline 5 years & 165 & 80.4 & 1.47 & 0.273 \\
\hline \multicolumn{5}{|l|}{ Fatigue } \\
\hline Baseline & 199 & 10.3 & 1.17 & \\
\hline 3 months & 205 & 12.2 & 1.09 & \\
\hline 1 year & 194 & 11.0 & 1.07 & \\
\hline 2 & 192 & 12.1 & 1.15 & \\
\hline 3 & 186 & 12.8 & 1.16 & \\
\hline 5 years & 166 & 12.5 & 1.22 & 0.328 \\
\hline \multicolumn{5}{|c|}{ Nausea and vomiting } \\
\hline Baseline & 199 & 1.3 & 0.47 & \\
\hline 3 months & 205 & 0.7 & 0.25 & \\
\hline 1 year & 195 & 0.5 & 0.20 & \\
\hline 2 & 192 & 1.2 & 0.40 & \\
\hline 3 & 186 & 1.0 & 0.35 & \\
\hline 5 years & 166 & 2.3 & 0.76 & 0.074 \\
\hline \multicolumn{5}{|l|}{ Pain } \\
\hline Baseline & 199 & 9.5 & 1.38 & \\
\hline 3 months & 207 & 7.5 & 1.19 & \\
\hline 1 year & 196 & 7.1 & 1.22 & \\
\hline 2 & 193 & 7.7 & 1.13 & \\
\hline 3 & 186 & 6.5 & 1.14 & \\
\hline 5 years & 166 & 7.7 & 1.24 & 0.482 \\
\hline \multicolumn{5}{|l|}{ Single item scales } \\
\hline \multicolumn{5}{|l|}{ Dyspnoea } \\
\hline Baseline & 198 & 12.1 & 1.51 & \\
\hline 3 months & 205 & 10.4 & 1.24 & \\
\hline 1 year & 195 & 12.5 & 1.37 & \\
\hline 2 & 192 & 12.2 & 1.38 & \\
\hline 3 & 184 & 12.3 & 1.50 & \\
\hline 5 years & 165 & 13.1 & 1.48 & 0.389 \\
\hline \multicolumn{5}{|l|}{ Sleep } \\
\hline Baseline & 199 & 17.3 & 1.81 & \\
\hline 3 months & 205 & 13.8 & 1.56 & \\
\hline 1 year & 195 & 13.7 & 1.67 & \\
\hline 2 & 192 & 13.7 & 1.72 & \\
\hline 3 & 186 & 14.3 & 1.60 & \\
\hline 5 years & 166 & 14.7 & 1.81 & 0.324 \\
\hline \multicolumn{5}{|l|}{ Appetite } \\
\hline Baseline & 199 & 2.5 & 1.71 & \\
\hline 3 months & 204 & 1.3 & 0.45 & \\
\hline
\end{tabular}




\begin{tabular}{|c|c|c|c|c|}
\hline QLQ C-30 Scale & $n^{a}$ & Mean & SEM & $\begin{array}{c}\text { p-Value } \\
\text { (ANOVA) }\end{array}$ \\
\hline 1 year & 195 & 1.9 & 0.55 & \\
\hline 2 & 192 & 3.0 & 0.81 & \\
\hline 3 & 186 & 1.8 & 0.61 & \\
\hline 5 years & 166 & 1.4 & 0.59 & 0.272 \\
\hline \multicolumn{5}{|l|}{ Constipation } \\
\hline Baseline & 199 & 2.8 & 0.85 & \\
\hline 3 months & 206 & 2.9 & 0.83 & \\
\hline 1 year & 196 & 4.1 & 0.95 & \\
\hline 2 & 193 & 2.2 & 0.94 & \\
\hline 3 & 186 & 3.6 & 0.91 & \\
\hline 5 years & 166 & 4.4 & 1.08 & 0.416 \\
\hline \multicolumn{5}{|l|}{ Diarrhoea } \\
\hline Baseline & 198 & 1.0 & 0.80 & \\
\hline 3 months & 206 & 4.3 & 0.88 & \\
\hline 1 year & 196 & 4.0 & 0.82 & \\
\hline 2 & 193 & 5.0 & 1.01 & \\
\hline 3 & 186 & 4.8 & 0.97 & \\
\hline 5 years & 166 & 3.8 & 1.12 & 0.894 \\
\hline \multicolumn{5}{|l|}{ Economy } \\
\hline Baseline & 195 & 1.0 & 0.48 & \\
\hline 3 months & 208 & 3.5 & 0.84 & $0.028^{*}$ \\
\hline 1 year & 196 & 2.4 & 0.70 & \\
\hline 2 & 191 & 1.6 & 0.51 & \\
\hline 3 & 186 & 2.2 & 0.66 & \\
\hline 5 years & 166 & 2.0 & 0.68 & $0.387^{*}$ \\
\hline PR-25 Scale & $\mathbf{N}$ & Mean & SEM & $\begin{array}{c}\text { p-Value } \\
\text { (ANOVA) }\end{array}$ \\
\hline \multicolumn{5}{|l|}{ Functional scales } \\
\hline \multicolumn{5}{|l|}{ Sexual activity } \\
\hline Baseline & 165 & 48.4 & 2.25 & \\
\hline 3 months & 189 & 43.4 & 3.36 & \\
\hline 1 year & 174 & 38.7 & 2.21 & \\
\hline 2 & 180 & 39.1 & 2.02 & \\
\hline 3 & 169 & 39.5 & 2.46 & \\
\hline 5 years & 149 & 40.4 & 4.26 & 0.252 \\
\hline \multicolumn{5}{|l|}{ Sexual functioning } \\
\hline Baseline & 124 & 84.1 & 1.67 & \\
\hline 3 months & 105 & 42.9 & 2.25 & $0.000^{*}$ \\
\hline 1 year & 102 & 47.6 & 2.86 & \\
\hline 2 & 112 & 50.6 & 2.34 & \\
\hline 3 & 103 & 50.2 & 2.50 & \\
\hline
\end{tabular}

\begin{tabular}{|c|c|c|c|c|}
\hline PR-25 Scale & $\mathbf{N}$ & Mean & SEM & $\begin{array}{c}\text { p-Value } \\
\text { (ANOVA) }\end{array}$ \\
\hline 5 years & 88 & 56.7 & 2.80 & $0.000^{*}$ \\
\hline \multicolumn{5}{|c|}{ Symptom scales } \\
\hline \multicolumn{5}{|c|}{ Urinary symptoms } \\
\hline Baseline & 170 & 17.1 & 1.26 & \\
\hline 3 months & 195 & 18.8 & 1.01 & \\
\hline 1 year & 183 & 15.1 & 0.92 & \\
\hline 2 & 186 & 14.8 & 1.05 & \\
\hline 3 & 176 & 15.6 & 1.23 & \\
\hline 5 years & 162 & 16.2 & 1.28 & $0.286^{*}$ \\
\hline \multicolumn{5}{|c|}{ Bowel symptoms } \\
\hline Baseline & 123 & 4.1 & 0.91 & \\
\hline 3 months & 166 & 2.1 & 0.38 & \\
\hline 1 year & 146 & 3.0 & 0.55 & \\
\hline 2 & 147 & 4.3 & 0.74 & \\
\hline 3 & 143 & 5.8 & 1.46 & \\
\hline 5 years & 132 & 4.5 & 0.91 & 0.057 \\
\hline \multicolumn{5}{|c|}{ Hormone treatment-related symptoms } \\
\hline Baseline & 166 & 6.2 & 0.72 & \\
\hline 3 months & 188 & 11.0 & 0.64 & $0.000 *$ \\
\hline 1 year & 176 & 11.8 & 0.91 & \\
\hline 2 & 179 & 11.1 & 0.85 & \\
\hline 3 & 173 & 11.8 & 0.86 & \\
\hline 5 years & 149 & 9.5 & 0.85 & $0.006^{*}$ \\
\hline \multicolumn{5}{|c|}{ Single item scale } \\
\hline \multicolumn{5}{|c|}{ Incontinence aid } \\
\hline Baseline & 10 & 13.3 & 5.44 & \\
\hline 3 months & 80 & 22.9 & 3.23 & \\
\hline 1 year & 43 & 17.1 & 4.20 & \\
\hline 2 & 39 & 17.1 & 3.21 & \\
\hline 3 & 40 & 11.7 & 3.28 & \\
\hline 5 years & 38 & 11.4 & 2.89 & 0.058 \\
\hline
\end{tabular}

*When significant ANOVA-test, the Bonferroni post hoc analysis was used. aInternal drop-outs: EORTC QLQ C-30, 1-26; PR 25, 27- 212.

functioning was rated high at baseline (mean 92.4) with a decrease at 3 months (mean 86.3) and a return to high social functioning at five years (mean 92.2, $\mathrm{p}=<0.003$ ).

QOL was rated at 78.1 points at baseline and lower after three months, 77.0. At five years, QOL had increased to an above-baseline rating, 80.4, though this increase was not significant $(\mathrm{p}=0.27)$.

Patients reported the least problems for fatigue (mean 10.3), nausea/vomiting (mean 1.3) and, pain at baseline. At five years, problems with nausea/vomiting and fatigue had 
increased, though not significantly. Problems with pain had slightly decreased at five years (ns).

In single-symptom scales for ratings of dyspnoea, sleep, appetite, constipation, diarrhoea and economy impact, most problems were scored for sleep (17.3) and dyspnoea (12.1), at baseline. At five years, ratings for sleeping problems had slightly decreased (ns) and for dyspnoea slightly increased (ns). Ratings for remaining single symptoms were still rated low at the 5-year assessment (ns).

\section{Prostate Cancer Specifics, PR 25}

Ratings for the PR-25 questionnaire (Table 2 cont.) for sexual activity showed a non-significant decrease over the five years, $48.4-40.4(\mathrm{p}=0.25)$, whereas sexual functioning decreased between baseline and 3 months $(p=<0.00)$ and was even lower at year five 56.7, $(\mathrm{p}=<0.00)$. Urinary problems scored the highest at baseline (17.1) and over time (16.2), and they did not decrease over time $(p=0.27)$. Bowel symptoms rated low at baseline and over time, and did not decrease over time, $4.1-4.5,(p=0.06)$.

Side effects from surgery, such as those after hormonal treatment, were rated 6.2 points at baseline and increased significantly both at three months $(11.0 \mathrm{p}=<0.00)$ and after five years $(9.5 \mathrm{p}=<0.00)$.

The use of incontinence aids showed an increase after three months, from 13.3 to 22.9 , and the increase had levelled out at year $5(\mathrm{p}=0.06)$.

\section{Influences on Quality of Life Over the Years from Functioning and Symptoms}

The multiple regression analysis (Tables $\mathbf{3}$ and $\mathbf{4}$ ) estimating influences on QOL from other variables in the C30 and PR-25 at five years showed significant values for nausea and vomiting $(\mathrm{p}=<0.00)$, emotional $(\mathrm{p}=<0.00)$ and social functioning $(p=<0.01)$ and pain $(p=<0.0 .02)$. All independent variables (functional and single items variables) were entered into the model at once, since the Pearsons' correlation showed correlations between 0.45-0.10. The results show how well the variables, as a set and individually, predict the effect on quality of life. No multicollinearity was found. Two outliers were identified with standardized residuals of 3.7 and 3.8 , respectively. Coook's distance showed no undue influence from these cases, 0.3, suggesting no large problem and they were thus not excluded. The model as a set explained $46.1 \%$ of the variance with a significance value of $\mathrm{p}=<0.00$

The impact on QOL from variables in the PR-25 (Table 5) showed that lack of hormone-related symptoms contributed significantly $(\mathrm{p}=<0.00)$ to QOL. The scales of bowel problems, PRBOW, and urinary aids, PRAID, had to be excluded to make a significant model. No multicollinearity was found between sexual activity, sexual functioning, urinary functioning and hormone-related symptoms. The model as a set explained $23.5 \%$ of the variance.

\section{Influence on Quality of Life Presented at Five Years}

The logistic regression (Table 5) was performed to answer the question of what problems were present after five years. Three independent areas were reported, emotional
Table 3. EORTC QLQ C-30 Functional and Single Item Variables Impact on Overall Quality of Life at 5 Years Following Treatment. Multiple Regression Analysis (n=162)

\begin{tabular}{|c|c|c|c|c|}
\hline \multirow{2}{*}{} & B & \multirow{2}{*}{$\mathbf{P}$} & \multicolumn{2}{|c|}{$\mathbf{9 5 \%}$ C-I ${ }^{\mathbf{1}}$ for B } \\
\cline { 4 - 5 } & & & Lower & Upper \\
\hline \hline PF & 0.11 & 0.21 & -0.10 & 0.44 \\
\hline RF & 0.00 & 0.99 & -0.25 & 0.23 \\
\hline EF & 0.27 & $<0.00^{*}$ & 0.16 & 0.59 \\
\hline CF & -0.05 & 0.51 & -0.32 & 0.15 \\
\hline SF & 0.24 & $<0.01 *$ & 0.11 & 0.48 \\
\hline FA & -0.10 & 0.26 & -0.35 & 0.09 \\
\hline NV & -0.29 & $<0.00^{*}$ & -0.86 & -0.29 \\
\hline PA & -0.18 & $<0.02^{*}$ & -0.41 & 0.04 \\
\hline DY & -0.14 & 0.07 & -0.30 & 0.01 \\
\hline SL & 0.14 & 0.51 & -0.00 & 0.24 \\
\hline AP & 0.08 & 0.25 & -0.15 & 0.53 \\
\hline CO & -0.11 & 0.12 & -0.32 & 0.04 \\
\hline DI & 0.04 & 0.60 & -0.15 & 0.25 \\
\hline FI & 0.10 & 0.16 & -0.08 & 0.50 \\
\hline
\end{tabular}

Dependent variable Quality of Life.

R Square $46.1 \%$.

C- ${ }^{1)}$ Confidence interval.

$*$ Sig. value $<0.05$.

Collinearity statistics, Tolerance $0.45-0.80$, VIF $1.52-2.25$.

Table 4. PR 25 Variables Impact on Quality of Life at Five Years Following Treatment. Multiple Regression Analysis $(\mathbf{n}=\mathbf{8 1})$

\begin{tabular}{|l|c|c|c|c|}
\hline & \multirow{2}{*}{ B } & P & \multicolumn{2}{|c|}{$\mathbf{9 5 \%}$ C-I $^{1)}$ for B } \\
\cline { 4 - 6 } & & & Lower & Upper \\
\hline \hline Sexual activity & 0.18 & 0.91 & -0.00 & 0.09 \\
\hline Sexual functioning & 0.04 & 0.72 & -0.17 & 0.17 \\
\hline Urinary functioning & -0.11 & 0.32 & -0.36 & 0.12 \\
\hline Hormone related symptoms & -0.38 & $<0.00 *$ & -0.90 & -0.20 \\
\hline
\end{tabular}

Dependent variable Quality of Life. Independent variables PRBOW, PRAID excluded due to dropouts.

R Square $23.5 \%$. Adjusted R for small sample $19.4 \%$.

${ }^{1)} \mathrm{C}$-I, Confidence interval.

*Sig. value $<0.05$.

Collinearity statistics, Tolerance $0.70-0.96$, VIF $1.42-1.04$.

$(p=<0.00)$, social functioning $(p=<0.00)$ and fatigue $(\mathrm{p}=<0.00)$. The model contained all 15 independent QLQ C30 variables (Table 3 ) and was statistically significant $\left(\chi^{2} 15\right.$, $\mathrm{n}=222$ ) $58.45, \mathrm{p}<0.00$, showing that the model was able to distinguish between early and late symptoms. The model explained 23.0\% (Cox and Snell $\mathrm{R}$ square) and 30.6\% (Nagelkerke $\mathrm{R}$ square) of the variance and correctly classified $75.0 \%$ of the cases. We noticed that there were no significant differences between baseline and five years in the ANOVA analyses for fatigue symptoms, while there were 
differences regarding emotional functioning as well as social functioning. For further correction, the goodness of fit test, Hosmer-Lemeshow test [16] was established with a $p$ value of 0.189 representing an acceptable model.

Table 5. Likelihood of Early vs Late Symptoms. Logistic Regression Analysis ( $0=$ Baseline, $1=5$ Years $)(n=222)$

\begin{tabular}{|c|c|c|c|c|c|}
\hline & \multirow{2}{*}{$\begin{array}{l}\text { Odds } \\
\text { Ratio }\end{array}$} & \multirow[t]{2}{*}{ Wald } & \multirow[t]{2}{*}{$\mathbf{p}$} & \multicolumn{2}{|c|}{$95 \% \mathrm{C}^{-I^{1)}}$ for Odds Ratio } \\
\hline & & & & Lower & Upper \\
\hline $\mathrm{PF}$ & 0.98 & 2.40 & 0.12 & 0.95 & 1.01 \\
\hline RF & 1.02 & 2.60 & 0.11 & 0.97 & 1.04 \\
\hline $\mathrm{EF}$ & 1.08 & 41.86 & $<0.00 *$ & 1.06 & 1.11 \\
\hline $\mathrm{CF}$ & 0.99 & 1.21 & 0.26 & 0.96 & 1.01 \\
\hline SF & 0.97 & 6.94 & $<0.00 *$ & 0.95 & 0.99 \\
\hline QL & 0.99 & 0.01 & 0.94 & 0.98 & 1.02 \\
\hline FA & 1.04 & 9.09 & $<0.00 *$ & 1.01 & 1.06 \\
\hline $\mathrm{NV}$ & 1.03 & 1.69 & 0.19 & 0.99 & 1.08 \\
\hline $\mathrm{PA}$ & 0.99 & 1.67 & 0.20 & 0.97 & 1.01 \\
\hline DY & 1.01 & 0.20 & 0.65 & 0.98 & 1.02 \\
\hline SL & 1.00 & 0.00 & 0.99 & 0.99 & 1.01 \\
\hline $\mathrm{AP}$ & 0.99 & 0.40 & 0.52 & 0.95 & 1.01 \\
\hline $\mathrm{CO}$ & 1.02 & 3.52 & 0.06 & 1.00 & 1.05 \\
\hline DI & 0.99 & 0.26 & 0.61 & 0.97 & 1.02 \\
\hline FI & 1.00 & 0.24 & 0.88 & 0.97 & 1.04 \\
\hline Constant & 0.07 & 1.81 & 0.18 & - & - \\
\hline
\end{tabular}

PF-FI categorical variables (EORTC QLQ C-30).

Hosmer and Lemeshow goodness-of-fit test, $\mathrm{p}=0.189$.

${ }^{1)} \mathrm{C}$-I, Confidence interval.

Cox \& Snell R square, 0.230, Nagelkerke R square, 0.306 .

$*$ Significant values $\mathrm{p}=<0.05$.

In the PR-25 module, none of the six scales showed any significant results in the multivariate analysis when tested with baseline $v s$ five years as the dependent variable. Goodness of fit was established by Hosmer-Lemelshow test with a $\mathrm{p}$ value of 0.292 representing an acceptable model. However, in the ANOVA analysis, sexual function and hormonal treatment-related symptoms showed significance i.e. more problems after five years. Adding variables such as living with a partner and employment status did not significantly affect the results concerning the variables in QLQ-30, nor the variables in the PR-25 module.

\section{DISCUSSION}

Our study investigated a population of patients with localized prostate cancer before and five years after retropubic prostatectomy. Patients represented a relatively young group of men (mean 62 years), considering that the mean age at the time of diagnosis in Sweden is 75, and this may have influenced the ratings positively. Younger men may be more prone to repeatedly complete questionnaires over a number of years. Huang determined that age at diagnosis, time from treatment and primary treatment were predictors of QOL in all treatments affecting urinary and sexual functions. In their study, problems occurred immediately after radical prostatectomy and returned to baseline after two years [20]. This fluctuation is not presented in our study, where problems remained and worsened over the years, i.e. regarding sexual functioning. The questionnaires are well-known and have shown high internal and external validity in a large number of studies. The QOL PR- 25 has been applied to prostate cancer study results for almost a decade now and is used as a cancerspecific complement to the EORTC QLQ C-30 questionnaire. Van Andel et al. stated discriminability between the two scales meeting criterion for construct validity [13]. It is to be noted there was a large rate of internal dropouts in the PR 25 variables. The reason for this is unknown to us and is not further looked into in this study. The phenomenon calls for further investigation.

There are some study limitations to be considered. Over the years, the patients were not followed in the study regarding further treatment and occurrence of co-morbidity. Whether or not everyday living was further affected by the prostate cancer and its treatment, or by other aspects from living, could not be established. However, quality of life was rated high, and problems stated after five years could reasonable be present in the wake of prostate cancer treatment or have other causes.

Patient-reported outcome of QOL did not differ over the five years of data collection. The result is comparable to other population studies that have shown similar ratings for QOL, e.g. Jakobsson, Lovén, Hallberg (2004) and Michelsson et al. (2000). In a study by Bach et al. [21], ratings for QOL decreased post-operatively, which they did not do in our study. Our study represented a somewhat younger group of men with a wider age range (36-75 years). Fransson et al. investigated patients given endocrine treatment with or without radiotherapy and found results comparable to ours and with no differences in quality of life after four years [22]. Endocrine treatment is otherwise known to affect bowel and sexual functioning negatively and radiotherapy is known to give bowel problems [23]. Patients in our study may have had endocrine or radiotherapy treatment which explains the reported problems from symptoms resembling hormonal treatment symptoms. The urinary and bowel problems also appear among men after $\mathrm{RP}$, which makes our study results comparable. Our results are also similar to results from QOL ratings from a normative sample of the Swedish population established by Michelson et al. [24]. Thus, it may be interpreted from our study results that prostate cancer is not significantly affecting QOL in physical aspects.

Emotional functioning showed low ratings at baseline and a significant increase over the five years. This is consistent with Bach et al. [21] who reported similar figures at baseline and an increase after surgery. Wallace et al. reported emotions from getting the diagnosis message in a qualitative study of prostate cancer survivors as 'shock', 'body blow', 'fear' and 'anger', emotions that may well be reactions in connection with the presentation of a cancer diagnosis [25]. Jakobsson et al. [26] presented similar results among cancer survivors, and it is reasonable to assume that patients in our study do not differ from other men in the same situation, and this would explain the increased ratings of emotional functioning after treatment and over the years. 
Social functioning decreased at three months and increased over the years. This may be an effect of postsurgery conditions in general, together with cathetertreatment side effects that are known to make patients stay at home and withdraw from social life during the treatment period [27]. The high physical, role, and cognitive functioning ratings over the five years with no fluctuations, may be connected to the men's age and good physical condition in general. It may be concluded that the men managed to handle the impact from surgery in a way that seemed not to adversely affect their daily life in these aspects.

Most single individual item symptoms did not change after surgery, and the highest scores, representing most problems, were found for sleeping problems and dyspnoea. Ratings did not decrease over time and the origin of the problems may not primarily be surgery or cancer, but connected to other problems. Thus, our results are similar to those of Fransson et al. [22], who found increased problems after four years among men who had external beam radiotherapy. They also found that emotional functioning and dyspnoea mostly affected QOL.

In a study by Erickson and Berger (2011) on patients with non-specified cancer, old age and anxiety were reasons for sleeping disorders. However, these problems may be present in a general population at times. Cancer-related symptoms, apart from anxiety, such as pain, cough and dyspnoea, as well as treatment-related factors, are described [28] as being similar to those for patients in our study. The connection between sleeping disorder and dyspnoea may be due to coughing and shortness of breath, as well as stemming from malignant disorders, and this study is not conclusive on this issue. Gupta et al. (2007) found that dyspnoea was a strong predictor for quality of life in patients with different cancer types, regardless of patient age and treatment [29]. This finding is not similar to our results. Gupta et al. suggest a longitudinal study design to confirm the association over time. Our study, over five years, shows lower ratings of dyspnoea at baseline than in that of Gupta et al. and displayed no difference in ratings over time.

Even though fatigue is a well-known adverse consequence of cancer and cancer treatment reported by Fransson and Gupta et al. for example [6, 29], initial scoring from our study showed lower figures for fatigue than for sleeping and dyspnoea problems. In the multivariate analyses, fatigue was not found to make a significant impact on QOL after five years. Whether fatigue was due to the cancer disease or not is not stated here.

Ratings of sexual activity and functioning from 3 months onwards decreased significantly post-surgery and decreased further in year five. A waning sexual functioning after prostate cancer treatment is in line with earlier study results and may be due to surgery techniques and post-surgery per se [30]. With regards to the relatively young age distribution of patients, it is surprising to find that sexual activity and functioning are not closer to baseline scores after five years. Also surprising is that sexual activity results did not decrease while sexual functioning did. This may be due to side effects from surgical techniques of nerve sparing but is also unexpected considering all modern pharmacological and technical aids on the market. Patients may be reluctant to give a truthful answer or to overestimate their self-rated ability. The highest internal dropout rates are in sexual and urinary areas of the questionnaires. Considering that no sexual functioning variables showed up significantly in the multivariate analyses, it is reasonable to assume that a sexual life was regarded less important than having survived the cancer disease.

Urinary problems and the use of incontinence aids increased in three months and decreased to baseline levels in five years, probably linked to a period of reduced catheter treatment and fewer leaking problems [30].

Clinically, the findings of this study call for nurses to better assess and focus on physical health problems of cancer patients when they have undertaken surgery and at follow up visits they need to pay more attention to emotional and social aspects of life, in addition to caring for pain, fatigue, nausea, and vomiting resulting from the surgery. Patients need information on problems that are present at that time and may not even remember having been given information if it was not called for. To be able to make a difference in the patient's post-surgical life, nurses need the ability to bridge the gap between in-hospital treatment and everyday life. Inhospital nurses may therefore need to make an extra effort in reporting patient status and life situation to community care nurses and not just the medical status in purpose to meet patients' needs.

\section{CONCLUSIONS}

QLQ areas mostly affected after surgery, at five years in relation to baseline, were positive effects on emotional and social functioning. The most outstanding impacts on QOL after five years were emotional and social functioning and lack of fatigue and nausea and vomiting. Most likely affected QOL ratings, though in positive way, were aspects of increased emotional and social functioning and lack of fatigue.

Sexual activity and functioning decreased after surgery and were not regained over the years, but did not negatively affect QOL. Emotional functioning and sexual activity presented a 10-point increase and decrease, respectively, and are therefore clinically most interesting according to our suggested interpretation. Problems were not increasing with increasing age, or whether living with a partner or not. The overall interpretation is that symptoms of illness and posttreatment shortcomings differed over time, but they did not affect QOL in a longitudinal perspective. It was interpreted that psychological aspects of life, such as emotional and social functioning, bridged physical shortcomings and contributed to a high quality of life. The finding supports and legitimizes the theoretical framework underpinning the study.

\section{CONFLICT OF INTEREST}

The authors confirm that they have no conflict of interest in regard to this manuscript.

\section{ACKNOWLEDGEMENTS}

Declared none. 


\section{REFERENCES}

[1] Cancer Incidence in Sweden. The Swedish National Board of Health and Wellfare. Official Statistics of Sweden. Stockholm: Centre for Epimemiology 2011.

[2] Cancerfonden, Socialstyrelsen. Cancer i siffror (Cancer in Figures). Stockholm 2009.

[3] Bill-Axelsson A, Holmberg L, Ruutu M, et al. Radical prostatectomy versus watchful waiting in early prostate cancer. $\mathrm{N}$ Engl J Med 2011; 364: 1708-17.

[4] Shunichi N, Shigeto I, Akihiro I, et al. Quality of life after radical prostatectomy in Japanese men: A 5-year follow up study. Int J Urol 2009; 16: 75-81.

[5] Ward-Smith P. Quality versus Quantity of life - What is the appropriate treatment outcome? Urol Nurs 2011; 31:375-6.

[6] Fransson P. Fatigue in prostate cancer patients treated with external beam radiotherapy: A prospective 5-year long-term patientreported evaluation. J Cancer Res Ther 2010; 6: 516-20.

[7] Jakobsson L, Loven L, Hallberg IR. Micturition problems in relation to quality of life in men with prostate cancer or benign prostatic hyperplasia: comparison with men from the general population. Cancer Nurs 2004; 27: 218-29.

[8] Stenholm S, Kronholm E, Sainio P, et al. Sleep-related factors and mobility in older men and women. J Gerontol A Biol Sci Med Sci 2010; 65: 649-57.

[9] Aaronson NK, Ahmedzai S, Bergman B, et al. The European Organisation for Research and Treatment of Cancer QLQ C-30: A quality-of-life instrument for use in international clinical trials in oncology. J Natl Cancer Inst 1993; 85: 365-76.

[10] Jakobsson L, Persson L, Lundqvist P. Daily life and life quality 3 years following prostate cancer treatment. BMC Nurs 2013; 12: 11.

[11] Abraham N, Makarov D, Laze J, Stefaovics E, Desai R, Lepor H. Patient centered outcomes in prostate cancer treatment: predictors of satisfaction up to 2 years after open radical retropubic prostatectomy. J Urol 2010; 184: 1977-81.

[12] Johansson E, Steineck G, Holmberg L, et al. Long-term quality-oflife outcome after radical prostectomy or watchful waiting: the Scandinavian Prostate cancer Group-4 randomised trial. Lancet Oncol 2011; 12: 891-9.

[13] van Andel G, Bottomley A, Fosså SD, et al. An international field study of the EORTC QLQ-PR25: A questionnaire for assessing the health-related quality of life of patients with prostate cancer. Eur $\mathrm{J}$ Cancer 2008; 44: 2418-24.

[14] Osoba D. What has been learned from measuring health-related quality of life in clinical oncology. Eur J Cancer 1999; 35: 156579.

[15] Fayers PM, Aaronson NK, Bjordal K, Groenvold M, Curran D, Bottomly A. The EORTC QLQ-C30 Scoring Manual 3rd Ed.
European Organisation for Research and Treatment of Cancer 2001.

[16] Altman DG. Practical Statistics for Medical Research. 7 ed. London: Chapman \& Hall 1997.

[17] Warner RM. Applied Statistics. From Bivariate Through Multivariate Techniques USA: Sage Publications Inc. 2008.

[18] Pallant J. SPSS. Survival Manual. $4^{\text {th }}$ ed. England: McGraw-Hill Education 2010.

[19] Polit DE, Beck CT. Nursing research. $8^{\text {th }}$ ed. Philadelphia: Lippincott, Williams and Wilkins 2012.

[20] Huang GJ, Sadetsky N, Penson DF. Health Related quality of life for men treated for localized prostate cancer with long-term followup. J Urol 2010; 183: 2206-12.

[21] Bach P, Döring T, Gesenberg A, Möhring C, Goepel M. Quality of life after retropubic prostatectomy - Pre- and postoperative scores of the EORTC QLQ C-30 and QLQ PR25. Health Qual Life Outcomes 2011; 9: 93

[22] Fransson P, Lund J-Å, Damber J-E, et al. Quality of life in patients with locally advanced prostate cancer given endocrine treatment with or without radiotherapy: 4 year follow-up of SPCG-7/SFU-3, an open-label, randomised, phase III trial. Lancet Oncol 2009; 10(4): 370-80.

[23] Stensvold A, Dahl A, Brennhovd B, et al. Bother problems in prostate cancer patients after curative treatment. Urol Oncol 2012; 31(7): 1067-78.

[24] Michelson H, Bolund C, Nilsson B, Brandberg Y. Health-related Quality of Life Measured by the EORTC QLQ-C30 ReferenceValues From a Large Sample of the Swedish Population. Acta Oncol 2000; 39: 477-84.

[25] Wallace KM, Burke M, Sanda MG, Hardy J, Bailey DE. The experiences of unpartnered men with prostate cancer: a qualitative analysis. J Cancer Surv 2011: 132-41.

[26] Jakobsson L, Hallberg IR, Loven L. Met and unmet nursing care needs in men with prostate cancer. An explorative study. Part II. Eur J Cancer Care 1997; 6: 117-23.

[27] Ezer H, Rigol Chchamovich J, Saad F, Aprikian A, Souhami L. Psychosocial adjustment of men during the first year of prostate cancer. Cancer Nurs 2012; 35: 141-7.

[28] Erickson J, Berger AM. Sleep-wake disturbances. Clin J Oncol Nurs 2011; 15: 123-7.

[29] Gupta D, Lis CG, Grutsch JF. The relationship between cancerrelated fatigue and patient satisfaction with quality of life in cancer. J Pain Symptom Manage 2007; 34: 40-7.

[30] Jakobsson L. Indwelling catheter treatment and health-related quality of life in men with prostate cancer in comparison with men with benign prostatic hyperplasia. Scand J Caring Sci 2002; 16: 264-71.

CC Jakobsson and Fransson; Licensee Bentham Open.

This is an open access article licensed under the terms of the Creative Commons Attribution Non-Commercial License (http://creativecommons.org/licenses/by-nc/3.0/) which permits unrestricted, non-commercial use, distribution and reproduction in any medium, provided the work is properly cited. 